http://dx.doi.org/10.18359/ravi.2685

\title{
Habilidades de liderazgo para una cultura de innovación en la gerencia de las universidades del Distrito de Santa Marta ${ }^{1}$
}

Sugey Martha Issa Fontalvo ${ }^{2}$

\author{
Servicio Nacional de Aprendizaje (SENA), Colombia ${ }^{3}$
}

Recibido, septiembre 16 de 2016

Concepto evaluación, noviembre 15 de 2016

Aceptado, diciembre 27 de 2016
Referencia: Issa Fontalvo, S. (2017). "Habilidades de liderazgo para una cultura de innovación en la gerencia de las universidades del Distrito de Santa Marta". Revista Academia y Virtualidad, 10, (1), 56-67

\section{Resumen}

Este artículo es producto de una investigación cuyo propósito fundamental fue analizar las habilidades del liderazgo para una cultura de innovación en la gerencia de las universidades del Distrito de Santa Marta. Para ello se estudiaron las bases teóricas de Horth y Buchner; Hargreaves y Fink; Argyris; Drucker y Rodríguez. La metodología utilizada fue el paradigma de investigación positivista, no experimental, transeccional, descriptiva y de campo. La población finita estuvo constituida por 37 empleados. Como instrumento para la recolección de datos se elaboró un cuestionario utilizando categorías de respuestas tipo Likert de 18 ítems, validado por siete expertos en el área. La confiabilidad del instrumento fue de 0.96 , determinada con el método Alfa de Cronbach. Los resultados indican la ausencia de habilidades del liderazgo. Asimismo, se concluye que la gerencia no posee capacidad para percibir aspectos y hechos relevantes, carece del manejo reflexivo de situaciones complejas, además de presentar problemas de comunicación. Finalmente, se recomienda elaborar talleres o cursos de formación sobre habilidades para lograr un cambio en el comportamiento en pos de un pensamiento estratégico innovador.

Palabras clave: habilidades de liderazgo, cultura de innovación, gerencia, comportamiento, pensamiento estratégico.

\footnotetext{
${ }^{1}$ Artículo de Investigación Científica, derivado del proyecto asociado "Cultura de Innovación para el desarrollo endógeno de la región en la gerencia de las universidades del Distrito de Santa Marta".

${ }^{2}$ Ingeniera de Sistemas; Especialista en Docencia Universitaria; Magíster en Telemática; PhD en Ciencias, mención Gerencia; Líder SENNOVA (Sistema de Investigación, desarrollo tecnológico e innovación). sugeyissa@hotmail.com.

${ }^{3}$ Centro de Logística y Promoción Ecoturística del Magdalena.
} 


\title{
Leadership skills to innovation culture related to management of universities at Santa Marta District
}

\begin{abstract}
This paper is a result of a research whose main goal is analyzing leadership skills for an innovation culture regarding managerial of universities at Santa Marta District. For this purpose, we implemented theories by Horth and Buchner; Hargreaves and Fink; Argyris; Drucker and Rodriguez. The research paradigm methodology was a positivist, non-experimental, crosssectional, descriptive and field focus, working with a finite population of 37 employees. Regarding data collection, a questionnaire was developed using Likert scale by 18 -item reply categories, validated by seven experts of this realm. The instrument reliability was 0.96 , established using the Cronbach Alpha method. Results indicate the management lacking leadership skills. Likewise, our conclusion: it is not able to perceive relevant aspects and facts, lacking the reflexive management of complex situations, besides communication breakdown. Finally, workshops or training courses may be recommended to develop skills in order to achieve a behavior change towards innovative strategic thinking.
\end{abstract}

Keywords: leadership skills, innovation culture, management, behavior, strategic thinking.

\section{Habilidades de liderança para uma cultura de inovação na gerência das universidades do Distrito de Santa Marta}

\section{Resumo}

Este artigo é produto de uma pesquisa cujo propósito fundamental foi analisar as habilidades de liderança para uma cultura de inovação na gerência das universidades do Distrito de Santas Marta. Para isso foram estudadas as bases teóricas de Horth e Buchner; Hargreaves e Fink; Argyris; Drucker e Rodríguez. A metodologia utilizada foi o paradigma de pesquisa positivista, não experimental, transeccional, descritiva e de campo. A população finita esteve constituída por 37 empregados. Como instrumento para a recolecção de dados elaborou-se um questionário utilizando categorias de respostas tipo Likert de 18 itens, validado por sete expertos na área. A confiabilidade do instrumento foi de 0,96 , determinada com o método Alfa de Cronbach. Os resultados indicam a ausência de habilidades de liderança. Assim mesmo conclui-se que a gerência não possui capacidade para perceber aspetos e fatos relevantes, carece do manejo em situações complexas, além de apresentar problemas de comunicação. Finalmente, recomenda-se a elaboração de oficinas ou cursos de formação sobre habilidades para atingir uma mudança no comportamento que leve a um pensamento estratégico inovador.

Palavras chave: habilidades de liderança, cultura da inovação, gerência, comportamento, pensamento estratégico. 


\section{Introducción}

Elmencionado fenómeno de la globalización ha dado origen al incremento de la comunicación entre los países trayendo como consecuencia la fusión de mercados, sociedades y culturas a partir de una serie de transformaciones sociales, económicas y políticas que les dan un carácter global. En este sentido, la cultura organizacional y la cultura de innovación son dos conceptos estrechamente relacionados, que se complementan mediante los procesos de comunicación, para determinar el éxito que puedan lograr las organizaciones. En tanto que la cultura organizacional hace referencia al conjunto de creencias, tradiciones, valores y principios compartidos que determinan el comportamiento de los miembros de una organización, la cultura de innovación se relaciona con la forma de pensar de los miembros de una organización como consecuencia del impulso de ideas para generar cambios o transformaciones a fin de obtener la eficiencia de la misma.

Por lo anterior y teniendo en cuenta que en todo el mundo existe un ambiente globalizado y competitivo, es clave que las organizaciones tengan una cultura organizacional fuerte, saludable y que apoye su estrategia, de tal manera que los empleados puedan desempeñar sus tareas con alta productividad. No cabe duda que tener una cultura de innovación constituye una fuerza poderosa que ha permitido a muchas pequeñas empresas trascender $\mathrm{y}$ convertirse con el paso del tiempo en grandes multinacionales que abarcan gran parte del territorio mundial.

De esta manera, las diferentes estrategias nacionales deben estar orientadas, por una parte, a la consolidación de capacidades básicas de investigación, desarrollo e innovación, formación de recursos humanos y generación de entornos favorables para la difusión de la ciencia y la tecnología a escala social con el fin de promover el desarrollo endógeno.

A medida que pasa el tiempo, cambian los modos de gestionar los procesos dentro de las organizaciones. La información y el conocimiento se califican como dos aspectos fundamentales que sobrellevan a una era innovadora, lo cual hace que las instituciones modernas se acoplen a un mercado competitivo, generador de nuevos adelantos y atentos a nuevos y múltiples desarrollos.

La innovación se plantea como una necesidad para que los territorios emerjan y sean competitivos. Las instituciones de educación superior no deben volver su mirada ante esta necesidad, sino por el contrario, emigrar en busca de acciones que promuevan el cambio y contribuyan al desarrollo de las localidades a partir de los nuevos efectos que se produzcan en satisfacción a las exigencias de esta sociedad moderna.

Contemplar la innovación en una institución de educación superior no debe ser un reto sino una acción cotidiana de transformación de la sociedad a partir de la gestión del conocimiento y de la participación de los distintos actores involucrados en la generación de estrategias que potencien las capacidades internas de la región.

En Colombia, el surgimiento de la globalización, junto a la gran dinámica generada por la sociedad de la información y del conocimiento, ha influido en la transformación en la estructura de la sociedad, dando lugar a nuevas formas de organización política, social, económica y cultural. En este sentido, cabe destacar que se deben establecer estrategias orientadas a generar capacidades científicas y tecnológicas comunes, y las instituciones educativas deben contemplar entornos para el establecimiento de redes y emprendimientos en el ámbito científico, tecnológico y de innovación, es decir, una cultura de innovación.

Este artículo representa la investigación que se llevó a cabo con la finalidad de analizar las habilidades del liderazgo para una cultura de innovación en la gerencia de las universidades del Distrito de Santa Marta. En este sentido, en principio se describe la fundamentación teórica, la orientación metodológica para la investigación constituida por el enfoque metodológico, la orientación en la tipología de la investigación y la validez y la confiabilidad del instrumento de recolección de la 
información; y finalmente se presentan los resultados de la investigación, las conclusiones y las referencias bibliográficas sobre las que se apoya el artículo para llevar a cabo la investigación.

\section{Fundamentación teórica}

Koontz y Weihrich (2007) definen el liderazgo como el arte o el proceso de influir en las personas para que se esfuercen voluntaria y entusiastamente en el cumplimiento de metas grupales. Mientras que para los autores Davis y Newstrom (2003), el liderazgo es la función ejercida por un adalid de una organización, que consiste en influir en los miembros para que persigan voluntariamente objetivos definidos, preferiblemente con entusiasmo.

En palabras de los autores Lussier y Achua (2016), el liderazgo es un proceso en el cual influyen líderes sobre seguidores, y viceversa, para lograr los objetivos de una organización a partir del cambio; los buenos líderes enseñan habilidades de liderazgo a su personal; quienes son líderes eficaces enseñan a los seguidores a pensar en función de la empresa, no de sí mismos.

Por su parte, Morcillo (2007) señala que la cultura de innovación es una forma de pensar $\mathrm{y}$ de actuar que genera, desarrolla y establece valores y actitudes en las organizaciones, tendientes a suscitar, asumir e impulsar ideas y cambios que supongan transformación, en el funcionamiento y eficiencia de la misma, aun cuando ello implique una ruptura con lo convencional o tradicional.

No obstante, la eficiencia y eficacia de un modelo de cultura depende del grado de consenso existente entre los valores, convicciones y patrones de comportamiento adoptados por los diferentes miembros y los grupos sociales que componen la organización.

\section{Habilidades del liderazgo para una cultura de innovación}

Horth y Buchner (2014) han identificado seis habilidades de pensamiento innovador; ellos señalan que, mediante el uso de estas capacidades, las organizaciones pueden crear algo útil y deseado, bien se trate de una tecnología puntera, de un servicio valioso o de una nueva solución a un viejo problema. Cada una de estas capacidades cambia la comprensión de una situación y abre la puerta a nuevos enfoques y soluciones.

\section{Prestar atención}

Hargreaves y Fink (2008) consideran que los líderes en organizaciones de educación superior con cultura de innovación, preservan, protegen y fomentan el aprendizaje profundo y amplio para todos, en unas relaciones de atención a los demás. En este sentido, las primeras impresiones y suposiciones no conforman la imagen total y por ello no conducen a una evaluación ajustada o a la mejor solución. Por prestar atención se entiende la capacidad de darse cuenta de algo que ha pasado desapercibido. Se trata de examinar una situación en mayor profundidad, de ser un observador agudo, de percibir los detalles e identificar nuevos patrones.

Para prestar atención es necesario frenar la marcha temporalmente a fin de ser reflexivo a la hora de comprender la situación. Es necesario entonces evaluar distintos puntos de vista y múltiples datos. $Y$ en esa medida resulta necesario observar y escuchar desde una nueva perspectiva, señalan Horth y Buchner (2014), lo que significa que el liderazgo en innovación en las organizaciones se centra en la percepción más aguda, más allá de las primeras impresiones.

\section{Personalizar}

En palabras de Hargreaves y Fink (2008), el liderazgo sostenible perdura, conserva y mejora los aspectos más valiosos del aprendizaje y la vida a lo largo del tiempo, año tras año, de un líder al siguiente. Asimismo, en el trabajo se tiende a minusvalorar la experiencia individual. Mediante la personalización se otorga más importancia a esta experiencia con el fin de extraer conclusiones de la misma. En el ámbito del pensamiento innovador, 
personalizar es un proceso doble, que consiste en aprovechar los amplios conocimientos y experiencias personales y en comprender al consumidor de forma personal y profunda.

Horth y Buchner (2014), al respecto, afirman que la capacidad de aprovechar experiencias y emociones personales sin relación aparente introduce perspectivas nuevas en los retos. La personalización aprovecha los intereses, pasatiempos o aficiones y los aplica al trabajo. Los autores establecen que la gerencia debe considerar de qué modo pueden contribuir las ideas, los patrones o las experiencias de toda una vida al trabajo de la organización. Por ejemplo, un directivo podría encontrar útil su experiencia como músico para ayudarle a orquestar y comunicar los diversos ritmos e intensidades del desarrollo de un producto a largo plazo por parte de su equipo. Del mismo modo se puede afirmar que la dimensión de la personalización que tiene que ver con el consumidor es la capacidad de comprenderle total y verdaderamente. ¿A quiénes se dirigen? ¿Qué les importa? ¿Qué desconocen sobre ellos? Personalizar requiere la interacción con los clientes en su entorno y empuja al directivo a comprender quiénes son y cómo viven estos clientes. El conocimiento profundo de los mismos conduce a ideas, patrones y experiencias nuevas que alimentan la innovación. Esto significa que en la gerencia de una organización, el liderazgo en innovación utiliza las experiencias personales para obtener nuevos puntos de vista.

\section{Imaginar}

El liderazgo sostenible no perjudica su entorno sino que lo mejora activamente, y para ello busca formas de compartir los conocimientos y los recursos con los centros educativos vecinos y la comunidad local, señalan Hargreaves y Fink (2008). Asimismo, Argyris (2005) afirma que con base en la creatividad, en un sistema complejo se puede poner en práctica la diversidad ya que éste puede regular el comportamiento para desarrollar elementos de la personalidad a fin de hacerla crítica y reflexiva, a la vez que innovadora y creadora, contribuyendo en esa medida a las comunidades humanas, las cuales poseen sus valores.

Esta capacidad de imaginación sirve para procesar la información. Las palabras, por sí solas, no bastan para extraer el sentido de informaciones complejas o muy vastas. Imaginar es un modo apropiado de abordar esta información y darle sentido. Las imágenes, historias, impresiones y metáforas son herramientas poderosas para describir situaciones, construir ideas y comunicar de manera eficaz. Utilizar la imaginación para responder a la pregunta ¿Qué pasaría si...? puede conducir a imágenes y posibilidades extraordinarias, afirman Horth y Buchner (2014). En este sentido, se infiere que el liderazgo en innovación genera información utilizando la metáfora.

\section{Jugar de manera reflexiva}

Drucker (2002) exploró cómo los líderes se organizaban en todos los sectores de la sociedad, señalando que existen siete fuentes principales de la innovación, a saber:

1. Lo inesperado, la sorpresa: una oportunidad surge de un hecho inesperado que puede tener su origen en un éxito o en un fracaso sorpresivo.

2. Lo incongruente: una incongruencia es una disonancia entre lo que es y lo que todos suponen que es. Es generalmente, un hecho que se refleja cualitativamente, puesto que en numerosas ocasiones pasa desapercibido.

3. Necesidad del proceso: aceptación total de que debe haber una mejor manera de hacer las cosas. Definición clara de las especificaciones para la solución correcta. No basta con sentir que algo se puede mejorar; debe ser posible definirlo $\mathrm{y}$ especificarlo.

4. Estructura del mercado $y$ del sector industrial: en esta instancia, hay que preguntarse: ¿en qué negocio se está? ¿Ha cambiado la estructura de la industria? Hay dos indicadores básicos que permiten detectar cuándo está cambiando la 
estructura del mercado: El sector industrial crece más rápido que la economía, y hay convergencia de tecnologías que modificarán sustancialmente el mapa de competencia.

5. Cambios demográficos: la demografía es un factor que hay que considerar determinante en el momento de decidir qué comprar, quién deberá comprarlo y en qué cantidad.

6. Cambios en la percepción: qué piensa la gente.

7. Los nuevos conocimientos: este segmento no sólo se basa en grandes conocimientos científicos o técnicos sino también en conocimientos sociales.

Otra característica de las innovaciones basadas en conocimientos es la convergencia. Estas innovaciones casi nunca se fundamentan en un solo factor, sino en la unión de varias categorías de ellos, afirma el autor.

Por su parte, Horth y Buchner (2014) afirman que el pensamiento del líder y el trabajo rutinario pueden convertirse en un proceso rígido. La innovación requiere romper algunas reglas, crecer y divertirse. Cuando se genera conocimiento y experiencia mediante formas no tradicionales, como la libre exploración, la improvisación, la experimentación, la construcción de prototipos de manera rápida y ligera o la exploración de los límites, el trabajo parece un juego pero los resultados que se obtienen son serios. Esto quiere decir que el liderazgo en innovación crea enfoques mediante la exploración y la experimentación.

\section{Indagar en colaboración con otras personas}

Hargreaves y Fink (2008), a su vez, consideran que esta dimensión corresponde a la habilidad de estimular la diversidad, aprende de ella y crea la cohesión y la interconexión entre sus componentes, ricos por su propia variedad. Horth y Buchner (2014), en su perspectiva, consideran que las innovaciones muy pocas veces son obra de un "genio solitario", sino que se obtienen a través de un intercambio de ideas reflexivo e imparcial.

Dentro de las habilidades del liderazgo para una cultura de innovación, resulta importante destacar los comportamientos relacionados con el proceso de transmitir información, para lograr la innovación al igual que escuchar las ideas, sugerencias y opiniones de sus empleados, consultando decisiones, ofreciendo y solicitando retroalimentación. Rodríguez (2008).

En este grupo se contemplan, asimismo, los procesos relacionados con la transmisión de información, es decir, escuchar ideas, sugerencias y opiniones de empleados, consultar decisiones, brindar y recibir retroalimentación que es una condición esencial para la innovación. Se infiere, entonces, que en el comportamiento gerencial hacia la innovación, los gerentes:

- Consultan sin egoísmo a los empleados idóneos, cuando se va a tomar una decisión.

- Facilitan el intercambio de tecnología e información.

- Cooperan para la innovación brindando la participación activa en proyectos de innovación compartidos con otras organizaciones.

- Facilitan la comunicación y fomentan la apertura para cuestionar sobre comportamientos que no han sido los más acertados.

- Dicen siempre lo que esperan de sus empleados para que los empleados sean creativos.

- Emiten sus juicios con claridad.

- Escuchan propuestas de mejoramiento de procesos y productos sin antes desecharlas. Desde las perspectivas de Horth y Buchner (2014), la indagación en colaboración consiste en un proceso de diálogo sostenido y eficaz con las partes interesadas. La gerencia debe aprovechar entonces la oportunidad que supone contar con múltiples partes interesadas y puntos de vista, puede generar una mayor complejidad, pero también es fuente de grandes oportunidades. Así, se debe plantear preguntas que inviten a la indagación, recurriendo al pensamiento crítico sin esperar respuestas inmediatas. En este sentido, se infiere que el liderazgo en innovación fomenta el dialogo productivo abarcando diversos puntos de vista. 


\section{Conciliación}

Esta habilidad, según Hargreaves y Fink (2008), trata de desarrollar los recursos humanos y materiales, no de reducirlos; de renovar la energía de las personas con prudencia y con iniciativa. La innovación requiere desechar el pensamiento exclusivo y contemplar el todo como una oposición inclusiva y abierta a una tercera (o cuarta, o quinta) solución.

Afirma Rodríguez (2008), al respecto, que dentro de las habilidades del liderazgo en el manejo de conflictos se incluyen los comportamientos relacionados con la manera en que los gerentes manejan sus propios errores y fracasos y los de sus empleados. También el manejo de problemas y la manera como aplican disciplina a sus empleados. Esto significa que el gerente que adopta una actitud en su desempeño:

- Da ejemplo de honestidad, puntualidad, sinceridad, cordialidad y creatividad.

- Acepta las fallas y equivocaciones y en ningún momento culpan a otros por ser más innovadores.

- Da oportunidad al diálogo escuchando las ideas o propuestas.

- Maneja retroalimentación de carácter positivo.

- Permite la libre expresión.

- Dinamiza los procesos de innovación, diálogo, integración y sostenimiento en todas las áreas de su empresa.

En palabras de Horth y Buchner (2014), conciliar permite a la gerencia vivir con la paradoja y la contradicción, y resolverlas. A diferencia del análisis tradicional del pensamiento empresarial, que requiere la división de los problemas en partes separadas, hechos conocidos y suposiciones actuales, conciliar tiene que ver con la síntesis, la integración y la posibilidad. Mediante lo que se conoce como razonamiento inductivo, la gerencia puede establecer conexiones intuitivas entre informaciones aparentemente no relacionadas y comenzar a extraer orden del caos, afirman los autores. En este sentido, el liderazgo en innovación realiza síntesis más que análisis (Horth y Buchner, 2014).

\section{Marco metodológico}

La metodología utilizada fue bajo el paradigma de investigación positivista, el tipo de investigación descriptiva, el diseño no experimental, transeccional y de campo. La población finita estuvo constituida por 37 empleados. Como instrumento para la recolección de datos se elaboró un cuestionario utilizando las categorías de respuestas de tipo Likert de 18 ítems, validado por siete expertos en el área.

La confiabilidad del instrumento fue determinada con el método Alfa de Cronbach presentando un 0.96 de confiabilidad. Posteriormente, se aplicó a la población del estudio constituida por las universidades del Distrito de Santa Marta. Finalmente se agrupan los datos haciendo uso de la estadística descriptiva (spss) construyendo las tablas de frecuencia, y posterior análisis e interpretación de los datos obtenidos.

\section{Resultados de la investigación}

Al analizar las habilidades del liderazgo para una cultura de innovación en la gerencia de las universidades del Distrito de Santa Marta, la evidencia presentada en la tabla 1 es el resultado de la identificación de las habilidades del liderazgo para una cultura de motivación en la gerencia de la población objeto de estudio. 
Tabla 1. Habilidades de liderazgo

\begin{tabular}{|c|c|c|c|c|c|c|c|c|c|c|c|c|c|c|}
\hline \multirow{2}{*}{$\begin{array}{l}\text { Categoría de } \\
\text { Respuesta }\end{array}$} & \multicolumn{2}{|c|}{ Atención } & \multicolumn{2}{|c|}{ Personalizar } & \multicolumn{2}{|c|}{ Imaginar } & \multicolumn{2}{|c|}{ Jugar } & \multicolumn{2}{|c|}{ Indagar } & \multicolumn{2}{|c|}{ Conciliar } & \multirow{2}{*}{$\mathrm{X}$} & \multirow{2}{*}{$\%$} \\
\hline & $\mathrm{Fi}$ & $\%$ & $\mathrm{Fi}$ & $\%$ & $\mathrm{Fi}$ & $\%$ & $\mathrm{Fi}$ & $\%$ & $\mathrm{Fi}$ & $\%$ & $\mathrm{Fi}$ & $\%$ & & \\
\hline Muy de Acuerdo & 7 & 19 & 6 & 17 & 6 & 17 & 7 & 18 & 5 & 13 & 8 & 22 & 7 & 18 \\
\hline De acuerdo & 9 & 23 & 9 & 23 & 8 & 22 & 7 & 19 & 5 & 14 & 7 & 18 & 7 & 20 \\
\hline $\begin{array}{l}\text { Ni de acuerdo, ni } \\
\text { en desacuerdo }\end{array}$ & 1 & 4 & 1 & 4 & 2 & 5 & 2 & 6 & 3 & 8 & 2 & 6 & 2 & 6 \\
\hline En desacuerdo & 10 & 28 & 12 & 32 & 11 & 29 & 12 & 32 & 13 & 36 & 11 & 30 & 12 & 31 \\
\hline Muy en desacuerdo & 10 & 26 & 9 & 23 & 10 & 27 & 9 & 26 & 11 & 29 & 9 & 24 & 10 & 26 \\
\hline Total & 37 & 100 & 37 & 100 & 37 & 100 & 37 & 100 & 37 & 100 & 37 & 100 & 37 & 100 \\
\hline
\end{tabular}

Los resultados reflejados en la tabla 1 permiten evidenciar que 10 de los sujetos que representan el $28 \%$ de los encuestados seleccionaron la categoría de respuesta negativa "En desacuerdo" sobre las afirmaciones contenidas en el cuestionario en función de la habilidad de prestar atención. De igual forma, 10 de los sujetos que representan el $26 \%$ de los encuestados seleccionaron la categoría de respuesta "Muy en desacuerdo"; 9 de los sujetos que representan el $23 \%$ de los encuestados seleccionaron la categoría de respuesta positiva "De acuerdo"; 7 de los sujetos que representan el $19 \%$ de los encuestados seleccionaron la categoría de respuesta positiva "Muy de acuerdo"; solo 1 de los sujetos que representa el $4 \%$ de los encuestados mantuvo una posición neutral al seleccionar la categoría de respuesta " $\mathrm{Ni}$ acuerdo, ni en desacuerdo".

En lo que se refiere a la habilidad de personalizar las experiencias, la tabla 1 refleja que 12 de los sujetos que representan el 32\% de los encuestados seleccionaron la categoría de respuesta negativa "En desacuerdo", 9 de los sujetos que representan el 23\% de los encuestados seleccionaron la categoría de respuesta "Muy en desacuerdo", 9 de los sujetos que representan el $23 \%$ de los encuestados seleccionaron la categoría de respuesta positiva "De acuerdo", 6 de los sujetos que representan el $17 \%$ de los encuestados seleccionaron la categoría de respuesta "Muy de acuerdo"; 1 de los sujetos que representa el $4 \%$ de los encuestados mantuvo una posición neutral seleccionando la categoría de respuesta " $\mathrm{Ni}$ de acuerdo, ni en desacuerdo".

En lo que respecta a la habilidad de imaginar, se debe notar que 11 de los sujetos que representan el $29 \%$ de los encuestados seleccionaron la categoría de respuesta negativa "En desacuerdo" sobre las afirmaciones contenidas en el cuestionario para identificar la capacidad de imaginación en la gerencia; 10 de los sujetos que representan el $27 \%$ de los encuestados seleccionaron la categoría de respuesta negativa "Muy en desacuerdo; 8 de los sujetos que representan el $22 \%$ de los encuestados seleccionaron la categoría de respuesta positiva "De acuerdo", 6 de los sujetos que representan el $17 \%$ de los encuestados seleccionaron la categoría de respuesta "Muy de acuerdo"; 2 de los sujetos que representan el $5 \%$ de los encuestados seleccionaron la categoría de respuesta "Ni de acuerdo, ni en desacuerdo".

En este mismo orden de ideas, los resultados sobre la habilidad de jugar, enuncian que 12 de los sujetos que representan el $32 \%$ de los encuestados seleccionaron la categoría de respuesta negativa "En desacuerdo" ante las afirmaciones contenidas en el cuestionario sobre la habilidad de jugar de manera reflexiva por parte de la gerencia; 9 de los sujetos que representan el 25\% seleccionaron la categoría de respuesta "Muy en desacuerdo", 7 de los sujetos que representan el 19\% de los encuestados seleccionaron la categoría de respuesta positiva "De acuerdo", 7 de los sujetos que representan el $18 \%$ de los encuestados seleccionaron la categoría de respuesta "Muy de acuerdo"; y 2 de los sujetos que representan el $6 \%$ de los encuestados se mantienen al margen al seleccionar la categoría de respuesta "Ni de acuerdo, ni en desacuerdo". 
En lo que respecta a la habilidad de indagar, 13 de los sujetos que representan el $36 \%$ de los encuestados respondieron en forma negativa al seleccionar la categoría de respuesta "En desacuerdo", 11 de los sujetos que representan el 19\% de los encuestados seleccionaron la categoría de respuesta "Muy en desacuerdo"; 5 de los sujetos que representan el $14 \%$ de los encuestados seleccionaron la categoría de respuesta positiva "De acuerdo", 5 de los sujetos que representan el $13 \%$ de los encuestados seleccionaron la categoría de respuesta "Muy de acuerdo"; solo 3 de los sujetos que representan el $8 \%$ de las personas encuestadas seleccionaron la categoría de respuesta "Ni de acuerdo, ni en desacuerdo". Finalmente, al analizar los resultados sobre la presencia de la habilidad de conciliación en la gerencia, se debe notar que 11 de los sujetos que representan el $30 \%$ de los encuestados responden en forma negativa seleccionando la categoría de respuesta "En desacuerdo", 9 de los sujetos que representan el $24 \%$ de los encuestados seleccionan la categoría de respuesta "Muy en desacuerdo"; 8 de los sujetos que representan el $22 \%$ de los encuestados seleccionan la categoría de respuesta positiva "Muy de acuerdo", 7 de los sujetos que representan el 18\% de los encuestados seleccionan la categoría de respuesta "De acuerdo"; y solo 2 de los sujetos que representan el $6 \%$ de los encuestados seleccionan la categoría de respuesta " $\mathrm{Ni}$ de acuerdo, ni en desacuerdo".

De acuerdo con los resultados reflejados en la tabla 1, el promedio de la presencia de las habilidades del liderazgo en la gerencia muestra que 12 encuestados que representan el $31 \%$ y 10 encuestados que representan el $26 \%$ seleccionan las categorías de respuestas negativas "En desacuerdo" y "Muy en desacuerdo", respectivamente. No obstante, el 20 y el $18 \%$ de los sujetos encuestados seleccionan las categorías de respuestas positivas "De acuerdo" y "Muy de acuerdo" respectivamente".

Asimismo, tal como se puede visualizar en la tabla 1, en el promedio de la presencia de las habilidades del liderazgo para una cultura de innovación en la gerencia de las universidades del Distrito de Santa Marta, 7 de los sujetos que representan el $20 \%$ de los encuestados, y 7 que representan el $18 \%$ de los encuestados seleccionaron las categorías de respuestas positivas; no obstante 12 que representan el $31 \%$ y 10 que representan el $26 \%$ de los encuestados seleccionaron las categorías de respuestas negativas.

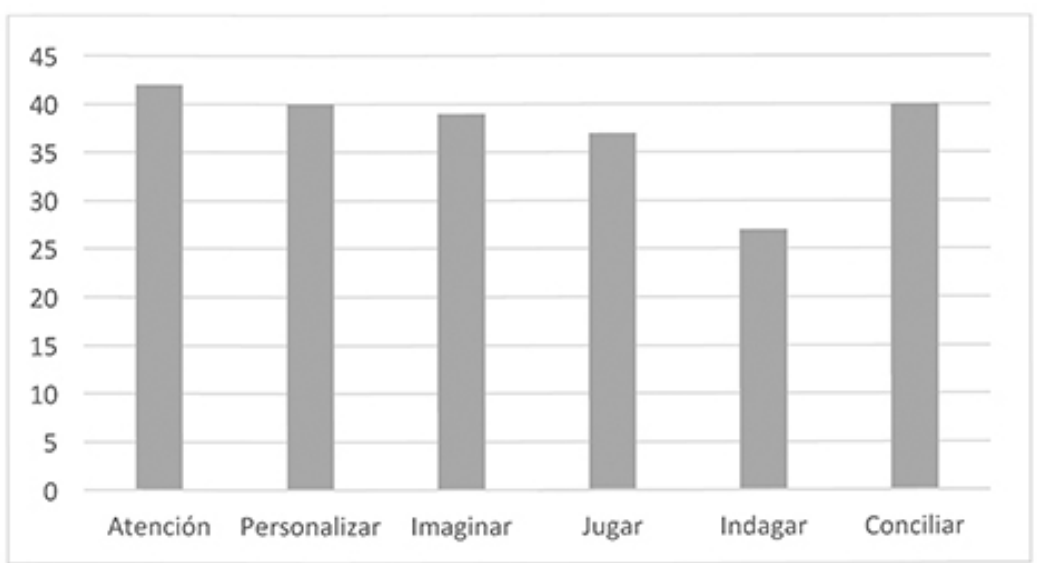

Figura 1. Habilidades de liderazgo. Fuente: Elaboración Propia (2016). 
La evidencia presentada en la figura 1 permite observar que, al sumar las categorías de respuestas positivas sobre la presencia de las habilidades del liderazgo en la gerencia, 16 de los sujetos que representan el $42 \%$ seleccionan las categorías de respuestas positivas, sobre la habilidad de prestar atención; 15 de los sujetos que representan el $40 \%$ seleccionaron las categorías de respuestas positivas al identificar la habilidad de personalizar; $15 \mathrm{de}$ los sujetos que representan el $40 \%$ consideran la habilidad de conciliación; 14 de los sujetos que representan el 39\% manifiestan la presencia de la habilidad de imaginación; 14 de los sujetos que representan el $37 \%$ aceptan la presencia de la habilidad de jugar y 10 sujetos que representan el $27 \%$ de los encuestados precisan la habilidad de indagación.

Del mismo modo, permite analizar las habilidades del liderazgo para una cultura de innovación en la gerencia de las universidades del Distrito de Santa Marta, no obstante, es importante mencionar que, en lo que se refiere a la habilidad de prestar atención, los autores Hargreaves y Fink (2008) expresan que se entiende como la capacidad de darse cuenta de algo que ha pasado desapercibido. Para prestar atención es necesario frenar la marcha temporalmente a fin de ser reflexivo a la hora de comprender la situación. Es necesario entonces evaluar distintos puntos de vista y múltiples datos.

En cuanto a la habilidad de personalizar, Hargreaves y Fink (2008) consideran que en el ámbito del pensamiento innovador, personalizar es un proceso doble, que consiste en aprovechar los amplios conocimientos y experiencias personales y en comprender al consumidor de forma personal y profunda. Para la habilidad de imaginación, Horth y Buchner (2014) plantean que es la capacidad que sirve de ayuda para procesar la información, pues imaginar es un buen modo de abordar esta información para darle sentido y como herramienta sirve para describir situaciones, construir ideas y comunicar de manera eficaz. Al hacer referencia sobre la habilidad de jugar de manera reflexiva, Drucker (2002) advierte que una oportunidad surge de un hecho inesperado que puede tener su origen en un éxito o en un fracaso sorpresivo. Por su parte, Horth y Buchner (2014) afirman que la innovación requiere romper algunas reglas, crecer y divertirse, y que el liderazgo en innovación crea enfoques mediante la exploración y la experimentación.

Con respecto a la habilidad de indagar en colaboración con otras personas, Hargreaves y Fink (2008) consideran que es la habilidad de estimular la diversidad, aprender de ella y crear la cohesión y la interconexión entre sus componentes, ricos por su propia variedad. De esta manera, Rodríguez (2008) afirma que, dentro de las habilidades del liderazgo para una cultura de innovación, es importante destacar los comportamientos relacionados con el proceso de transmitir información, a fin de lograr la innovación al igual que escuchar las ideas, sugerencias y opiniones de sus empleados, consultando decisiones, ofreciendo y solicitando retroalimentación.

En lo que se refiere a la habilidad de conciliación, Rodríguez (2008) establece que, dentro de las habilidades del liderazgo en el manejo de conflictos, se incluyen los comportamientos relacionados con la manera como los gerentes manejan sus propios errores y fracasos y los de sus empleados. Además, acepta las fallas y equivocaciones $y$ en ningún momento culpan a otros por ser más innovadores.

Los resultados indican que las habilidades del liderazgo para una cultura de innovación en la gerencia de las universidades del Distrito de Santa Marta reflejan porcentajes muy bajos, por lo que se concluye que para una mayoría significativa de los sujetos encuestados la gerencia no posee capacidad de darse cuenta que algo ha pasado desapercibido, carece del manejo de situaciones difíciles de manera reflexiva y no escucha propuestas de sus miembros para innovar, evidenciándose la ausencia de la habilidad de prestar atención.

Del mismo modo, los resultados obtenidos sobre la habilidad de personalizar permiten concluir en la gerencia, la ausencia de un pensamiento innovador con el que aprovecha las experiencias personales de sus miembros, 
del establecimiento de la interacción con los clientes en su entorno y del uso de las experiencias personales para obtener nuevos puntos de vista. Asimismo, los resultados indican que la mayoría de los sujetos encuestados niegan que la gerencia comparta recursos con la comunidad local, que sea innovadora para las comunidades humanas y que construya ideas para comunicarse de manera eficaz evidenciando la ausencia de la habilidad de imaginar en la gerencia.

En cuanto a la habilidad de jugar de manera reflexiva, vale anotar que una gran mayoría de los sujetos encuestados niegan que la gerencia para innovar algunas veces rompa reglas, crea enfoques mediante la exploración para la innovación y que considera que una oportunidad de innovación puede surgir de un hecho inesperado. Con respecto a la habilidad de indagar en colaboración con otras personas, los resultados reflejan que para una mayoría significativa de los sujetos encuestados, la gerencia de las universidades no estimula la diversidad, niega que las innovaciones se obtengan a través de un intercambio de ideas y no escucha las opiniones de sus empleados, consultando decisiones.

Finalmente, en lo que se refiere a la habilidad de conciliación, los resultados reflejan que para la mayoría de los sujetos encuestados es falso que la gerencia en ningún momento culpa a otros por ser más innovadores, que maneja sus propios errores y que es ejemplo de creatividad.

\section{Conclusiones}

Al identificar las habilidades del liderazgo para una cultura de innovación, los resultados están por debajo del $50 \%$, por lo que este estudio permite concluir que la gerencia de las universidades en estudio carece de habilidades para prestar atención, personalizar, imaginar, jugar de manera reflexiva, indagar en colaboración con otras personas y capacidad de conciliación. En este sentido, se concluye que la gerencia carece de capacidad para darse cuenta de algo que ha pasado desapercibido, comprender al consumidor de forma personal, crear enfoques mediante la experimentación, estimular la diversidad y manejar conflictos.

Cabe señalar que esta ausencia de una cultura de innovación puede acarrear una serie de peligros, pues sin ella, las organizaciones no pueden hacerle frente a la competitividad generada por el fenómeno de la globalización. En concreto, se pueden confundir las demandas sociales con los intereses empresariales y que realmente no lleguen a la sociedad conocimientos, productos o servicios que no se consideren rentables. Además, la ausencia de transferencia de recursos para investigación y desarrollo, dan origen a la desvinculación de los profesores a la práctica de su profesión, a la falta de motivación hacia la participación de los profesionales de la producción y los servicios en el proceso de formación de los profesionales afines.

Por lo anterior, resulta indispensable la ejecución de talleres o cursos de formación sobre habilidades gerenciales para una cultura de innovación con el fin de obtener cambios en el comportamiento gerencial hacia una gerencia con un pensamiento estratégico innovador que utiliza capacidades para el logro de tecnología de punta, de un servicio valioso o de una nueva solución a un viejo problema abriendo la puerta para nuevos enfoques y soluciones.

Asimismo, se considera necesario fortalecer la presencia de los aspectos de la cultura de innovación en la gerencia de las universidades del Distrito de Santa Marta, incrementando el aprendizaje organizacional al igual que el proceso dentro de la organización mediante el cual se desarrolla un conocimiento sobre la relación entre las acciones realizadas, los resultados obtenidos y los efectos que éstos tienen en el entorno.

De esta manera, al precisar los elementos de la cultura de innovación en la gerencia de las universidades del Distrito de Santa Marta, se recomienda la formación de un equipo directivo potente con gran sentido de autonomía, que fusione nuevos valores de gestión con los valores académicos tradicionales, de tal manera que todos los niveles de la institución trabajen para mejorar la cultura académica y 
propicien una cultura innovadora integrada que coopere con la participación activa en proyectos de innovación, e integre la creatividad para todos los niveles y los procesos.

\section{Referencias}

Argyris, Ch. (2001). Sobre el aprendizaje organizacional. Segunda Edición. México: Editorial Universidad Iberoamericana.

Davis, K. y Newstrom, J. (2003). Comportamiento humano en el trabajo. Décima Edición. México: Editorial McGrawHill Interamericana.

Drucker, P. (2002). Los desafíos de la gerencia para el siglo XXI. Bogotá: Norma. Hargreaves, A. y Fink, D. (2008). El liderazgo sostenible. Siete principios para el liderazgo en centros educativos innovadores. Madrid: Ediciones Morata.

Hargreaves, A. y Fink, D. (2008). El liderazgo sostenible. Siete principios para el liderazgo en centros educativos innovadores. Madrid: Ediciones Morata.

Horth, D. y Buchner, D. (2014). Liderazgo en materia de innovación. Cómo utilizar la innovación para liderar con eficacia, trabajar en colaboración y obtener resultados. Recuperado de http://www. ccl.org/wp-content/uploads/2015/04/ InnovationLeadership.pdf

Koontz, H. y Weihrich, H. (2007). Elementos de administración: Un enfoque internacional. Séptima Edición. México: Editorial McGrawHill Interamericana.

Lussier, R. y Achua, C. (2016). Liderazgo. Teoría, aplicación y desarrollo de habilidades. México: Cengage Learning Editores.

Morcillo, P. (2007). Cultura e innovación empresarial. Madrid: Ediciones Paraninfo.
Rodríguez, M. (2008). Formación gerencial en valores: conceptos y prácticas. Bogotá: Editorial Universidad Nacional de Colombia. 